\title{
Effects of Intensive Blood-Pressure Control in Type 2 Diabetes Mellitus
}

\section{The ACCORD Study Group ${ }^{\star}$}

\begin{abstract}
BACKGROUND—There is no evidence from randomized trials to support a strategy of lowering systolic blood pressure below 135 to $140 \mathrm{~mm} \mathrm{Hg}$ in persons with type 2 diabetes mellitus. We investigated whether therapy targeting normal systolic pressure (i.e., $<120 \mathrm{~mm} \mathrm{Hg}$ ) reduces major cardiovascular events in participants with type 2 diabetes at high risk for cardiovascular events.
\end{abstract}

METHODS-A total of 4733 participants with type 2 diabetes were randomly assigned to intensive therapy, targeting a systolic pressure of less than $120 \mathrm{~mm} \mathrm{Hg}$, or standard therapy, targeting a systolic pressure of less than $140 \mathrm{~mm} \mathrm{Hg}$. The primary composite outcome was nonfatal myocardial infarction, nonfatal stroke, or death from cardiovascular causes. The mean follow-up was 4.7 years.

RESULTS-After 1 year, the mean systolic blood pressure was $119.3 \mathrm{~mm} \mathrm{Hg}$ in the intensivetherapy group and $133.5 \mathrm{~mm} \mathrm{Hg}$ in the standard-therapy group. The annual rate of the primary outcome was $1.87 \%$ in the intensive-therapy group and $2.09 \%$ in the standard-therapy group (hazard ratio with intensive therapy, $0.88 ; 95 \%$ confidence interval $[\mathrm{CI}], 0.73$ to $1.06 ; \mathrm{P}=0.20$ ). The annual rates of death from any cause were $1.28 \%$ and $1.19 \%$ in the two groups, respectively (hazard ratio, $1.07 ; 95 \% \mathrm{CI}, 0.85$ to $1.35 ; \mathrm{P}=0.55$ ). The annual rates of stroke, a prespecified secondary outcome, were $0.32 \%$ and $0.53 \%$ in the two groups, respectively (hazard ratio, 0.59 ; $95 \% \mathrm{CI}, 0.39$ to $0.89 ; \mathrm{P}=0.01$ ). Serious adverse events attributed to antihypertensive treatment occurred in 77 of the 2362 participants in the intensive-therapy group (3.3\%) and 30 of the 2371 participants in the standard-therapy group $(1.3 \%)(\mathrm{P}<0.001)$.

CONCLUSIONS-In patients with type 2 diabetes at high risk for cardiovascular events, targeting a systolic blood pressure of less than $120 \mathrm{~mm} \mathrm{Hg}$, as compared with less than $140 \mathrm{~mm}$ $\mathrm{Hg}$, did not reduce the rate of a composite outcome of fatal and nonfatal major cardiovascular events. (ClinicalTrials.gov number, NCT00000620.)

\section{Copyright () 2010 Massachusetts Medical Society}

Address reprint requests to Dr. Cushman at the Preventive Medicine Section (111Q), Veterans Affairs Medical Center, 1030 Jefferson Ave., Memphis, TN 38104, or at william.cushman@va.gov.

The members of the Writing Group (William C. Cushman, M.D., Gregory W. Evans, M.A., Robert P. Byington, Ph.D., David C. Goff, Jr., M.D., Ph.D., Richard H. Grimm, Jr., M.D., Ph.D., Jeffrey A. Cutler, M.D., M.P.H., Denise G. Simons-Morton, M.D., Ph.D., Jan N. Basile, M.D., Marshall A. Corson, M.D., Jeffrey L. Probstfield, M.D., Lois Katz, M.D., Kevin A. Peterson, M.D., William T. Friedewald, M.D., John B. Buse, M.D., Ph.D., J. Thomas Bigger, M.D., Hertzel C. Gerstein, M.D., and Faramarz Ismail-Beigi, M.D., Ph.D.) assume responsibility for the integrity of the article.

${ }^{*}$ The members of the Action to Control Cardiovascular Risk in Diabetes (ACCORD) Study Group are listed in Section 1 in Supplementary Appendix 1, available with the full text of this article at NEJM.org. The affiliations of the members of the Writing Group are listed in the Appendix.

Disclosure forms provided by the authors are available with the full text of this article at NEJM.org. 
Diabetes mellitus increases the risk of cardiovascular disease by a factor of two to three at every level of systolic blood pressure. ${ }^{1}$ Because cardiovascular risk in patients with diabetes is graded and continuous across the entire range of levels of systolic blood pressure, even at prehypertensive levels, the Seventh Report of the Joint National Committee on Prevention, Detection, Evaluation, and Treatment of High Blood Pressure (JNC 7) recommended beginning drug treatment in patients with diabetes who have systolic blood pressures of 130 $\mathrm{mm} \mathrm{Hg}$ or higher, with a treatment goal of reducing systolic blood pressure to below 130 $\mathrm{mm} \mathrm{Hg} .{ }^{1-3}$ There is, however, a paucity of evidence from randomized clinical trials to support these recommendtions. The Action to Control Cardiovascular Risk in Diabetes (ACCORD) blood pressure trial (ACCORD BP) ${ }^{4}$ tested the effect of a target systolic blood pressure below $120 \mathrm{~mm} \mathrm{Hg}$ on major cardiovascular events among high-risk persons with type 2 diabetes. We present here the main results of the ACCORD BP trial.

\section{METHODS \\ STUDY DESIGN}

ACCORD was a randomized trial conducted at 77 clinical sites organized into seven networks in the United States and Canada (for a full list of participating institutions and investigators, see Section 1 in Supplementary Appendix 1, available with the full text of this article at NEJM.org). The trial enrolled 10,251 high-risk participants with type 2 diabetes mellitus. ${ }^{5}$ All participants were randomly assigned to either intensive or standard glycemic control (the ACCORD glycemia trial). In addition, 5518 of the ACCORD participants were also randomly assigned (in a 2-by-2 factorial design) to either simvastatin plus fenofibrate or simvastatin plus placebo (the ACCORD lipid trial), and the remaining 4733 participants were also randomly assigned (in a 2-by-2 factorial design) to either intensive or standard blood-pressure control (the ACCORD blood-pressure trial). Details of the randomization are provided in Section 3 of Supplementary Appendix 1. The trial was sponsored by the National Heart, Lung, and Blood Institute (NHLBI). The protocol was approved by the institutional review board or ethics committee at each center and by an independent protocol review committee appointed by the NHLBI. The main results of the ACCORD glycemia trial have been published previously, ${ }^{6}$ and the main results of the ACCORD Lipid trial are published elsewhere in this issue of the Journal. ${ }^{7}$ The ACCORD trial protocol and amendments are available in Supplementary Appendix 2.

\section{ELIGIBILITY CRITERIA AND RECRUITMENT}

Inclusion criteria for the glycemia trial are described in detail elsewhere. ${ }^{5}$ In brief, participants were eligible if they had type 2 diabetes mellitus and a glycated hemoglobin level of $7.5 \%$ or more and were 40 years of age or older with cardiovascular disease or 55 years of age or older with anatomical evidence of a substantial amount of atherosclerosis, albuminuria, left ventricular hypertrophy, or at least two additional risk factors for cardiovascular disease (dyslipidemia, hypertension, smoking, or obesity). Exclusion criteria included a body-mass index (the weight in kilograms divided by the square of the height in meters) of more than 45 , a serum creatinine level of more than $1.5 \mathrm{mg}$ per deciliter (132.6 $\mu \mathrm{mol}$ per liter), and other serious illness. Participants with a systolic blood pressure between 130 and $180 \mathrm{~mm} \mathrm{Hg}$ who were taking three or fewer antihypertensive medications and who 
had the equivalent of a 24 -hour protein excretion rate of less than $1.0 \mathrm{~g}$ were also eligible for the blood-pressure trial (see Section 4 in Supplementary Appendix 1). ${ }^{8}$ All participants provided written informed consent.

Recruitment occurred during two noncontiguous periods: 491 participants in the bloodpressure trial were recruited from January 2001 through early June 2001 during a "vanguard" phase, and the remaining 4242 participants were recruited from January 2003 through October 2005 during the main trial phase. An upper age limit of 79 years was added to the eligibility criteria for the main trial recruitment.

\section{TRIAL PROCEDURES}

The ACCORD BP trial was a nonblinded trial in which participants were randomly assigned to intensive therapy that targeted systolic blood pressures of less than $120 \mathrm{~mm} \mathrm{Hg}$ or standard therapy that targeted systolic blood pressures of less than $140 \mathrm{~mm} \mathrm{Hg}$. Treatment strategies that are currently available in clinical practice were used to lower blood pressure. Randomization was performed centrally on the study's Web site with the use of permuted blocks to maintain concealment of future study-group assignments.

The approach to the management of blood pressure has been described elsewhere. ${ }^{4}$ The schedules of visits for the assessment and management of blood pressure differed according to treatment group. For participants in the intensive-therapy group, visits to assess blood pressure were scheduled once a month for 4 months and every 2 months thereafter; for participants in the standard-therapy group, visits were scheduled at months 1 and 4 and every 4 months thereafter. Additional visits were scheduled as needed in both groups to monitor and ensure appropriate implementation of the study intervention strategies. In both blood-pressure groups, participants who were assigned to intensive glycemic therapy had more frequent contacts for the management of glycemia, but blood pressure was not monitored at these additional visits.

The ACCORD BP trial was a study of a treatment strategy to achieve specific systolic blood-pressure goals, rather than an evaluation of any specific drug regimen. However, all the antihypertensive regimens were to include drug classes that had been shown to result in a reduction in cardiovascular events among participants with diabetes. Details of the assessment of blood pressure, the adjustment of medication doses, and antihypertensive drug regimens are provided in Sections 8 and 9 in Supplementary Appendix 1. Antihypertensive drugs were donated by Abbott Laboratories, AstraZeneca Pharmaceuticals, GlaxoSmithKline Pharmaceuticals, King Pharmaceuticals, Sanofi-Aventis U.S., and Novartis Pharmaceuticals. Sphygmomanometers were donated by Omron Healthcare. The companies that donated the drugs and devices had no role in the design of the study, the accrual or analysis of the data, or the preparation of the manuscript.

At the 4-month visits that both treatment groups were scheduled to attend, information on study outcomes and adverse events was ascertained, blood samples were obtained, and clinical examinations were performed. The occurrence of self-reported symptoms of swelling or of dizziness on standing during the previous month was assessed as part of a standardized symptom checklist that was administered at baseline and at 1, 3, and 4 years 
after randomization to a random sample of 969 participants who were assessed for healthrelated quality of life.

\section{TRIAL OUTCOMES}

The primary outcome for all three ACCORD trials was the first occurrence of a major cardiovascular event, which was defined as the composite of nonfatal myocardial infarction, nonfatal stroke, or cardiovascular death. Prespecified secondary outcomes included the combination of the primary outcome plus revascularization or hospitalization for congestive heart failure (termed the "expanded macrovascular outcome"); the combination of a fatal coronary event, nonfatal myocardial infarction, or unstable angina (termed "major coronary disease events"); nonfatal myocardial infarction; fatal or nonfatal stroke; nonfatal stroke; death from any cause; death from cardiovascular causes; and hospitalization or death due to heart failure. Definitions of each prespecified end point and information regarding methods of ascertainment are included in Section 6 in Supplementary Appendix 1.

Since all the antihypertensive medications used in the trial were approved by the Food and Drug Administration and were used according to approved labeling, we limited detailed data collection on serious adverse events to those attributed by investigators to antihypertensive medications (see Section 7 in Supplementary Appendix 1). Clinical and laboratory variables, including serum potassium and creatinine levels and estimated glomerular filtration rate, ${ }^{9}$ were also examined as potential adverse effects.

\section{STATISTICAL ANALYSIS}

With a planned sample size of 4200 participants, the ACCORD BP trial was designed to have $94 \%$ power to detect a $20 \%$ reduction in the rate of the primary outcome for participants in the intensive-therapy group, as compared with those in the standard-therapy group, assuming a two-sided alpha level of 0.05 , a primary-outcome rate of $4 \%$ per year in the standard-therapy group, and a planned average follow-up of 5.6 years for participants who did not have an event. Since ACCORD was a factorially designed trial, the targeted number of participants and the determination of sample size were made under the assumption that the intensive glucose-lowering intervention would produce a $15 \%$ benefit. $^{5}$

Statistical analyses were conducted at the coordinating center with the use of S-Plus software, version 8.0 (Insightful) or SAS software, version 9.1 (SAS Institute). Baseline characteristics and key safety outcomes were compared between the two study groups with the use of the chi-square test, Fisher's exact test, Wilcoxon rank-sum test, and the twosample t-test.

Analyses of primary and secondary outcomes were performed with the use of time-to-event methods according to the intention-to-treat principle. Event rates are expressed as the percentage of events per follow-up year, taking into account the censoring of follow-up data. Kaplan-Meier estimates were used to calculate the proportion of participants who had an event during follow-up.

Occurrences of primary and secondary outcomes in the two study groups were compared with the use of hazard ratios and $95 \%$ confidence intervals. Two-sided $\mathrm{P}$ values were 
calculated with the use of likelihood-ratio tests from Cox proportional-hazards regression analyses. The Cox models contained a term representing study-group assignments plus terms accounting for the following prespecified stratifying variables: assignment to the intensive glucose-lowering intervention, each of the seven clinical-center networks, and the presence or absence of a previous cardiovascular event. Using the log of follow-up time as a time-dependent covariate, we found no evidence of important departures from the assumption of proportionality. ${ }^{10}$ We examined the consistency of the intervention effect on the primary outcome among nine prespecified subgroups using statistical tests of interaction between the treatment effect and the subgroup within the Cox models.

During the trial, an independent data and safety monitoring committee appointed by the NHLBI monitored the primary outcome (11 times) and total rate of death (7 times) with the use of O'Brien-Fleming boundaries determined by the Lan-DeMets approach. For these two outcomes, $\mathrm{P}$ values were adjusted to account for the number, timing, and results of interim analyses. All other $\mathrm{P}$ values for secondary outcomes and for subgroup analyses are nominal and have not been adjusted for multiple comparisons.

All analyses are based on observed data with the assumption that missing data were missing completely at random. For the longitudinal analysis of systolic blood pressure, a sensitivity analysis with the use of maximum-likelihood methods, under the assumption that the missing data were missing at random, is presented in Section 13 in Supplementary Appendix 1.

\section{RESULTS STUDY PARTICIPANTS}

A total of 4733 participants were enrolled in the ACCORD BP trial. Of these, 2362 were randomly assigned to intensive blood-pressure control and 2371 were assigned to standard therapy. Baseline characteristics were generally similar between the two groups (Table 1). The mean age of the participants was 62.2 years; $47.7 \%$ were women and $33.7 \%$ had cardiovascular disease at baseline. The mean systolic and diastolic blood pressures of the participants at baseline were $139.2 \mathrm{~mm} \mathrm{Hg}$ and $76.0 \mathrm{~mm} \mathrm{Hg}$, respectively.

At the end of the trial (June 2009), vital status was known for $95.1 \%$ of the randomly assigned participants. The mean duration of follow-up for the rate of death was 5.0 years, or $98.4 \%$ of the potential person-years of follow-up that would have been available if all surviving participants had been followed until the end of the trial. The mean duration of follow-up for the primary outcome was 4.7 years (94.8\% of the potential follow-up). At the final follow-up visit, the rate of current smoking was $8.5 \%$ in the intensive-therapy group and $7.5 \%$ in the standard-therapy group $(\mathrm{P}=0.44)$.

\section{BLOOD PRESSURE}

The two therapeutic strategies quickly resulted in different systolic blood-pressure levels (Fig. 1). After the first year of therapy, the average systolic blood pressure at the 4-month protocol visits that both groups attended was $119.3 \mathrm{~mm} \mathrm{Hg}$ (95\% confidence interval [CI], 118.9 to 119.7 ) in the intensive-therapy group and $133.5 \mathrm{~mm} \mathrm{Hg}$ (95\% CI, 133.1 to 133.8) in 
the standard-therapy group, resulting in an average between-group difference of $14.2 \mathrm{~mm}$ $\mathrm{Hg}$ (95\% CI, 13.7 to 14.7). The corresponding mean diastolic blood pressures were 64.4 (95\% CI, 64.1 to 64.7) and 70.5 (95\% CI, 70.2 to 70.8), for an average difference of $6.1 \mathrm{~mm}$ Hg (95\% CI, 5.7 to 6.5) (Section 14 in Supplementary Appendix 1).

The lower blood pressure in the intensive-therapy group was associated with a greater exposure to drugs from every class (Fig. 1, and Section 11 in Supplementary Appendix 1). The mean number of medications after the first year was 3.4 (95\% CI, 3.4 to 3.5) in the intensive-therapy group and 2.1 (95\% CI, 2.1 to 2.2$)$ in the standard-therapy group.

\section{ADVERSE EVENTS}

As compared with the standard-therapy group, the intensive-therapy group had significantly higher rates of serious adverse events attributed to antihypertensive treatment, as well as higher rates of hypokalemia and elevations in serum creatinine level (Table 2). The mean estimated glomerular filtration rates were significantly lower in the intensive-therapy group than in the standard-therapy group at the last visit. There were significantly more instances of an estimated glomerular filtration rate less than $30 \mathrm{ml}$ per minute per $1.73 \mathrm{~m}^{2}$ of bodysurface area in the intensive-therapy group than in the standard-therapy group (99 vs. 52 events, $\mathrm{P}<0.001$ ), although only 38 participants in the intensive-therapy group and 32 in the standard-therapy group had two or more instances of that rate $(\mathrm{P}=0.46)$. The frequency of macroalbuminuria at the final visit was significantly lower in the intensive-therapy group than in the standard-therapy group, and there was no between-group difference in the frequency of end-stage renal disease or the need for dialysis. In the random sample of 969 participants who were assessed for health-related quality of life, the frequency of symptoms of orthostatic hypotension was similar between the groups.

\section{CLINICAL OUTCOMES}

The primary composite outcome of nonfatal myocardial infarction, nonfatal stroke, or death from cardiovascular causes occurred in 445 participants. The rate was $1.87 \%$ per year in the intensive-therapy group as compared with 2.09\% per year in the standard-therapy group, with no significant between-group difference (hazard ratio with intensive therapy, $0.88 ; 95 \%$ CI, 0.73 to 1.06; $\mathrm{P}=0.20$ ) (Table 3 and Fig. 2).

There were 294 deaths from any cause and 118 deaths from cardiovascular causes (Table 3). Rates of death from any cause were $1.28 \%$ per year in the intensive-therapy group and $1.19 \%$ in the standard-therapy group (hazard ratio with intensive therapy, 1.07; 95\% CI, 0.85 to $1.35 ; \mathrm{P}=0.55$ ). Rates of death from cardiovascular causes were $0.52 \%$ per year in the intensive-therapy group and $0.49 \%$ in the standard-therapy group (hazard ratio, 1.06; $95 \% \mathrm{CI}, 0.74$ to $1.52 ; \mathrm{P}=0.74)$.

The two study groups did not differ significantly with respect to most of the other secondary outcomes. Nominally significant differences were seen in the rate of total stroke $(0.32 \%$ per year in the intensive-therapy group vs. $0.53 \%$ per year in the standard-therapy group; hazard ratio, $0.59 ; 95 \% \mathrm{CI}, 0.39$ to $0.89 ; \mathrm{P}=0.01)$ and in the rate of nonfatal stroke $(0.30 \%$ per year in the intensive-therapy group vs. $0.47 \%$ per year in the standard-therapy group; hazard 
ratio, $0.63 ; 95 \% \mathrm{CI}, 0.41$ to $0.96 ; \mathrm{P}=0.03$ ). There were no significant interactions among prespecified subgroups (see Section 17 in Supplementary Appendix 1).

\section{DISCUSSION}

Intensive antihypertensive therapy in the ACCORD BP trial did not significantly reduce the primary cardiovascular outcome or the rate of death from any cause, despite the fact that there was a significant and sustained difference between the intensive-therapy group and the standard-therapy group in mean systolic blood pressure. There was also no significant benefit with respect to most of the secondary trial outcomes. At a significance level of less than 0.05 , intensive blood-pressure management did reduce the rate of two closely correlated secondary outcomes - total stroke and nonfatal stroke. Assuming that this finding was real, the number needed to undergo intensive blood-pressure management to prevent one stroke over the course of 5 years was 89 . These effects would be consistent with the findings of two meta-analyses of the effect of a reduction of $10 \mathrm{~mm} \mathrm{Hg}$ in systolic blood pressure on the incidence of stroke ${ }^{11,12}$; the meta-analyses showed a relative risk with blood-pressure reduction of 0.64 with the use of data from observational studies and of 0.59 with the use of data from drug-treatment trials. ${ }^{12}$

The interpretation of the ACCORD BP results is complicated by the fact that the event rate observed in the standard-therapy group was almost 50\% lower than the expected rate. This result may have been a consequence of the frequent use of statins and of inclusion criteria that directed participants with dyslipidemia into the ACCORD lipid trial, leaving participants who were at lower risk in the blood-pressure trial. ${ }^{5}$ The reduced power was reflected in the relatively wide confidence interval that does not exclude a $27 \%$ benefit for the primary end point.

There were some signals of possible harm associated with intensive blood-pressure control, including a rate of serious adverse events that was significantly higher in the intensivetherapy group than in the standard-therapy group. Both the estimated glomerular filtration rate and macroalbuminuria were reduced, but the implications of these changes on cardiovascular and renal outcomes are uncertain.

The United Kingdom Prospective Diabetes Study ${ }^{13,14}$ and a post hoc subgroup analysis of the Hypertension Optimal Treatment (HOT) trial ${ }^{15}, 16$ showed reductions in cardiovascular events with antihypertensive therapy among patients with type 2 diabetes mellitus, but the participants in their intensively treated groups had much higher mean systolic bloodpressure levels (144 mm Hg in both cases) than did the participants in either group of our trial. In the Action in Diabetes and Vascular Disease: Preterax and Diamicron Modified Release Controlled Evaluation trial (ADVANCE; ClinicalTrials.gov number, NCT00145925), ${ }^{17}$ active treatment with an angiotensin-converting-enzyme inhibitor and a thiazide-type diuretic reduced the rate of death but did not significantly reduce a composite macrovascular outcome. However, the ADVANCE trial had no specified blood-pressure goals, and the mean systolic blood pressure in the intensive group (135 $\mathrm{mm} \mathrm{Hg}$ ) was not as low as the mean systolic blood pressure even in the ACCORD standard-therapy group. It is possible that lowering systolic blood pressure from the mid-130s to approximately $120 \mathrm{~mm}$ 
Hg does not further reduce most cardiovascular events or the rate of death, and most of the benefit from lowering blood pressure is achieved by targeting a goal of less than $140 \mathrm{~mm}$ $\mathrm{Hg}$. Alternatively, it is possible that 5 years is not long enough to see significant cardiac benefits from the normalization of systolic blood pressure among persons with diabetes who have good control of glycemia, especially when other effective treatments, such as statins and aspirin, are used frequently.

There are several limitations of the ACCORD BP trial. First, the trial had an open-label design, a design that was not likely to have affected blood-pressure goals or measurement or the blinded ascertainment of the outcomes but may have affected the reporting of adverse events; second, the rate of cardiovascular events was lower than the expected rate in the standard-therapy group; and third, patients younger than 40 years of age were not included in the study and patients older than 79 years of age were not included after the vanguard phase. In addition, although it was not the intent of this trial to test the blood-pressure goal of $130 \mathrm{~mm} \mathrm{Hg}$ that was recommended in the JNC 7 (a recommendation that was made after the ACCORD trial was initiated), it would be difficult to argue that such a target would be better than a target of $140 \mathrm{~mm} \mathrm{Hg}$, since even a blood-pressure goal of $120 \mathrm{~mm} \mathrm{Hg}$ did not confer benefit.

In conclusion, the ACCORD BP trial evaluated the effect of targeting a systolic blood pressure of $120 \mathrm{~mm} \mathrm{Hg}$, as compared with a goal of $140 \mathrm{~mm} \mathrm{Hg}$, among patients with type 2 diabetes at high risk for cardiovascular events. The results provide no evidence that the strategy of intensive blood-pressure control reduces the rate of a composite of major cardiovascular events in such patients.

\section{Acknowledgments}

Supported by contracts from the National Heart, Lung, and Blood Institute (N01-HC-95178, N01-HC-95179, N01HC-95180, N01-HC-95181, N01-HC-95182, N01-HC-95183, N01-HC-95184, and IAA\#Y1-HC-9035 and IAA\#Y1-HC-1010). Other components of the National Institutes of Health, including the National Institute of Diabetes and Digestive and Kidney Diseases, the National Institute on Aging, and the National Eye Institute, contributed funding. The Centers for Disease Control and Prevention funded substudies within ACCORD on costeffectiveness and health-related quality of life. General Clinical Research Centers provide support at many sites. The following companies provided study medications, equipment, or supplies: Abbott Laboratories, Amylin Pharmaceutical, AstraZeneca Pharmaceuticals, Bayer HealthCare, Closer Healthcare, GlaxoSmithKline Pharmaceuticals, King Pharmaceuticals, Merck, Novartis Pharmaceuticals, Novo Nordisk, Omron Healthcare, Sanofi-Aventis U.S., and Takeda.

\section{APPENDIX}

The affiliations of the ACCORD Blood Pressure writing group are as follows: Preventive Medicine Section, Memphis Veterans Affairs (VA) Medical Center, Memphis (W.C.C.); Division of Public Health Sciences, Wake Forest University School of Medicine, WinstonSalem, NC (G.W.E., R.P.B., D.C.G.); Berman Center for Outcomes and Clinical Research (R.H.G.), and Department of Family Practice and Community Health, University of Minnesota (K.A.P.) - both in Minneapolis; National Heart, Lung, and Blood Institute, Bethesda, MD (J.A.C., D.G.S.-M.); Primary Care, Ralph H. Johnson Veterans Affairs Medical Center, Charleston, SC (J.N.B.); Department of Medicine, University of Washington, Seattle (M.A.C., J.L.P.); VA New York Harbor Healthcare System (L.K.), 
Departments of Biostatistics and Epidemiology, Columbia University Mailman School of Public Health (W.T.F.), and Division of Cardiology, Columbia University College of Physicians and Surgeons (J.T.B.) — all in New York; Division of Endocrinology, University of North Carolina School of Medicine, Chapel Hill (J.B.B.); Department of Medicine and the Population Health Research Institute, McMaster University, Hamilton, ON, Canada (H.C.G.); and Departments of Medicine and Physiology and Biophysics, Case Western Reserve University, Cleveland (F.I.-B.).

\section{REFERENCES}

1. Stamler J, Vaccaro O, Neaton JD, Wentworth D. Diabetes, other risk factors, and 12 yr cardiovascular mortality for men screened in the Multiple Risk Factor Intervention Trial. Diabetes Care. 1993; 16:434-44. [PubMed: 8432214]

2. Adler AI, Stratton IM, Neil HA, et al. Association of systolic blood pressure with macrovascular and microvascular complications of type 2 diabetes (UKPDS 36): prospective observational study. BMJ. 2000; 321:412-419. [PubMed: 10938049]

3. Chobanian AV, Bakris GL, Black HR, et al. The Seventh Report of the Joint National Committee on Prevention, Detection, Evaluation, and Treatment of High Blood Pressure: the JNC 7 report. JAMA. 2003; 289:2560-2572. [PubMed: 12748199] Erratum, JAMA. 2003; 290:197.

4. Cushman W, Grimm RH Jr, Cutler JA, et al. Rationale and design for the blood pressure intervention of the Action to Control Cardiovascular Risk in Diabetes (ACCORD) trial. Am J Cardiol. 2007; 99(Suppl):44i-55i.

5. ACCORD Study Group. Action to Control Cardiovascular Risk in Diabetes (ACCORD) trial: design and methods. Am J Cardiol. 2007; 99(Suppl):21i-33i.

6. The Action to Control Cardiovascular Risk in Diabetes Study Group. Effects of intensive glucose lowering in type 2 diabetes. N Engl J Med. 2008; 358:2545-2559. [PubMed: 18539917]

7. The ACCORD Study Group. Effects of combination lipid therapy in type 2 diabetes mellitus. N Engl J Med. 2010; 362:1563-1574. [PubMed: 20228404]

8. Peterson JC, Adler S, Burkart JM, et al. Blood pressure control, proteinuria, and the progression of renal disease. Ann Intern Med. 1995; 123:754-762. [PubMed: 7574193]

9. Levey AS, Coresh J, Greene T, et al. Using standardized serum creatinine values in the Modification of Diet in Renal Disease study equation for estimating glomerular filtration rate. Ann Intern Med. 2006; 145:247-54. [PubMed: 16908915] Erratum, Ann Intern Med. 2008; 149:519.

10. Therneau, TM.; Grambsch, PM. Modeling survival data: extending the Cox model. New York: Springer-Verlag; 2000.

11. MacMahon S, Peto R, Cutler J, et al. Blood pressure, stroke, and coronary heart disease. Part 1. Prolonged differences in blood pressure: prospective observational studies corrected for the regression dilution bias. Lancet. 1990; 335:765-774. [PubMed: 1969518]

12. Law MR, Morris JK, Wald NJ. Use of blood pressure lowering drugs in the prevention of cardiovascular disease: metaanalysis of 147 randomised trials in the context of expectations from prospective epidemiological studies. BMJ. 2009; 338:b1665. [PubMed: 19454737]

13. UK Prospective Diabetes Study Group. Tight blood pressure control and risk of macrovascular and microvascular complications in type 2 diabetes: UKPDS 38. BMJ. 1998; 317:703-713. [PubMed: 9732337] Erratum, BMJ. 1999; 318:29.

14. Idem. Efficacy of atenolol and captopril in reducing risk of macrovascular and microvascular complications in type 2 diabetes: UKPDS 39. BMJ. 1998; 317:713-720. [PubMed: 9732338]

15. Hansson L, Zanchetti A, Carruthers SG, et al. Effects of intensive blood-pressure lowering and low-dose aspirin in patients with hypertension: principal results of the Hypertension Optimal Treatment (HOT) randomised trial. Lancet. 1998; 351:1755-1762. [PubMed: 9635947]

16. Zanchetti A, Hansson L, Clement D, et al. Benefits and risks of more intensive blood pressure lowering in hypertensive patients of the HOT study with different risk profiles: does a J-shaped curve exist in smokers? J Hypertens. 2003; 21:797-804. [PubMed: 12658027] 
17. Patel A, MacMahon S, Chalmers J, et al. Effects of a fixed combination of perindopril and indapamide on macrovascular and microvascular outcomes in patients with type 2 diabetes mellitus (the ADVANCE trial): a randomised controlled trial. Lancet. 2007; 370:829-840. [PubMed: 17765963] 


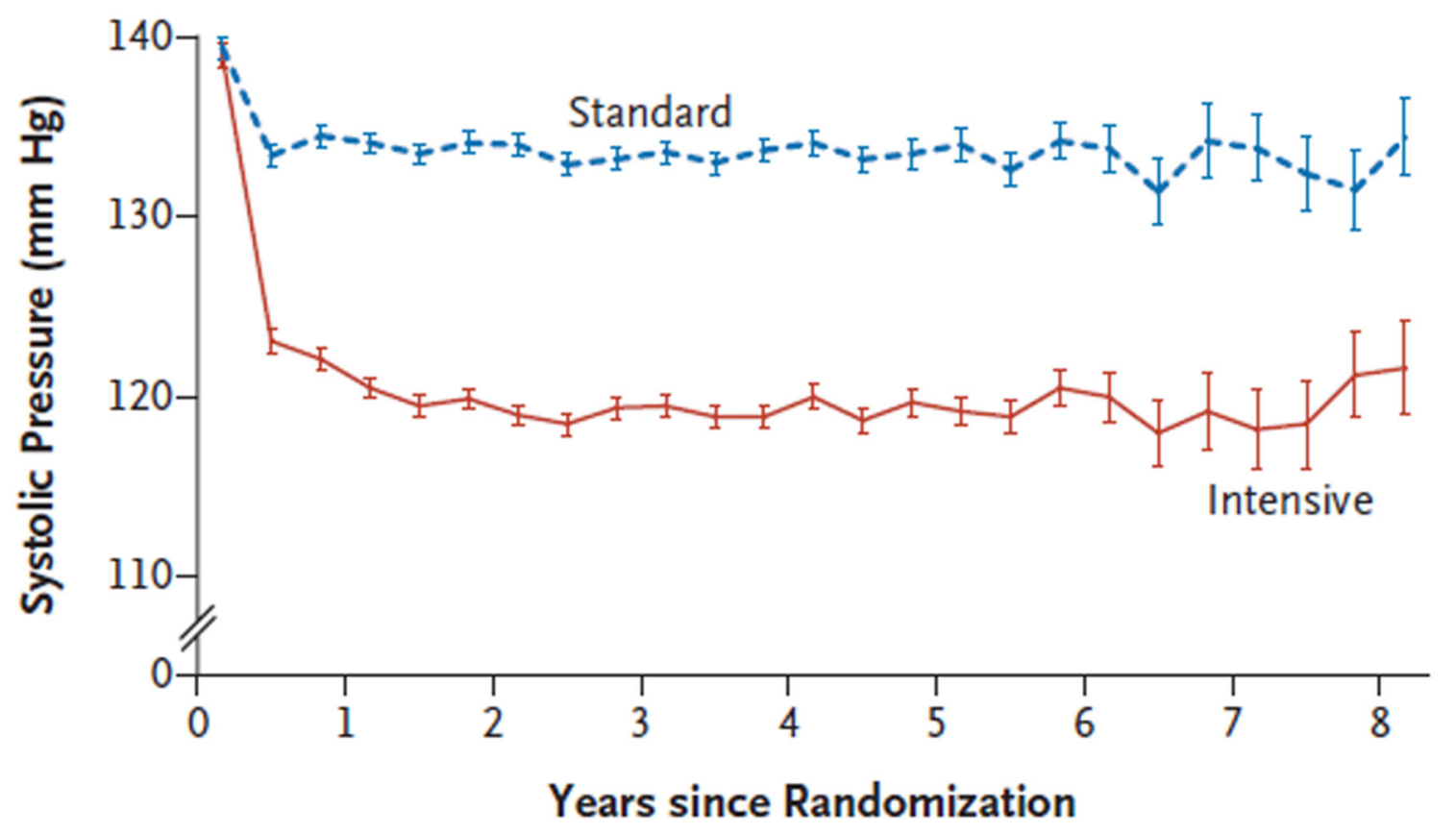

Mean No. of Medications

Prescribed

Intensive

3.2

3.4

3.4

3.5

3.5

3.5

3.4

3.4

Standard

$\begin{array}{ll}1.9 & 2.1\end{array}$

2.1

2.2

2.2

2.3

2.3

2.3

No. of Patients

Intensive

$2174 \quad 207$

$1973 \quad 1792$

1150

445

156

156

Standard $2208 \quad 2136$ 2077

$\begin{array}{ll}1860 & 1241\end{array}$

$504 \quad 203$

201

Figure 1. Mean Systolic Blood-Pressure Levels at Each Study Visit

I bars indicate $95 \%$ confidence intervals. 


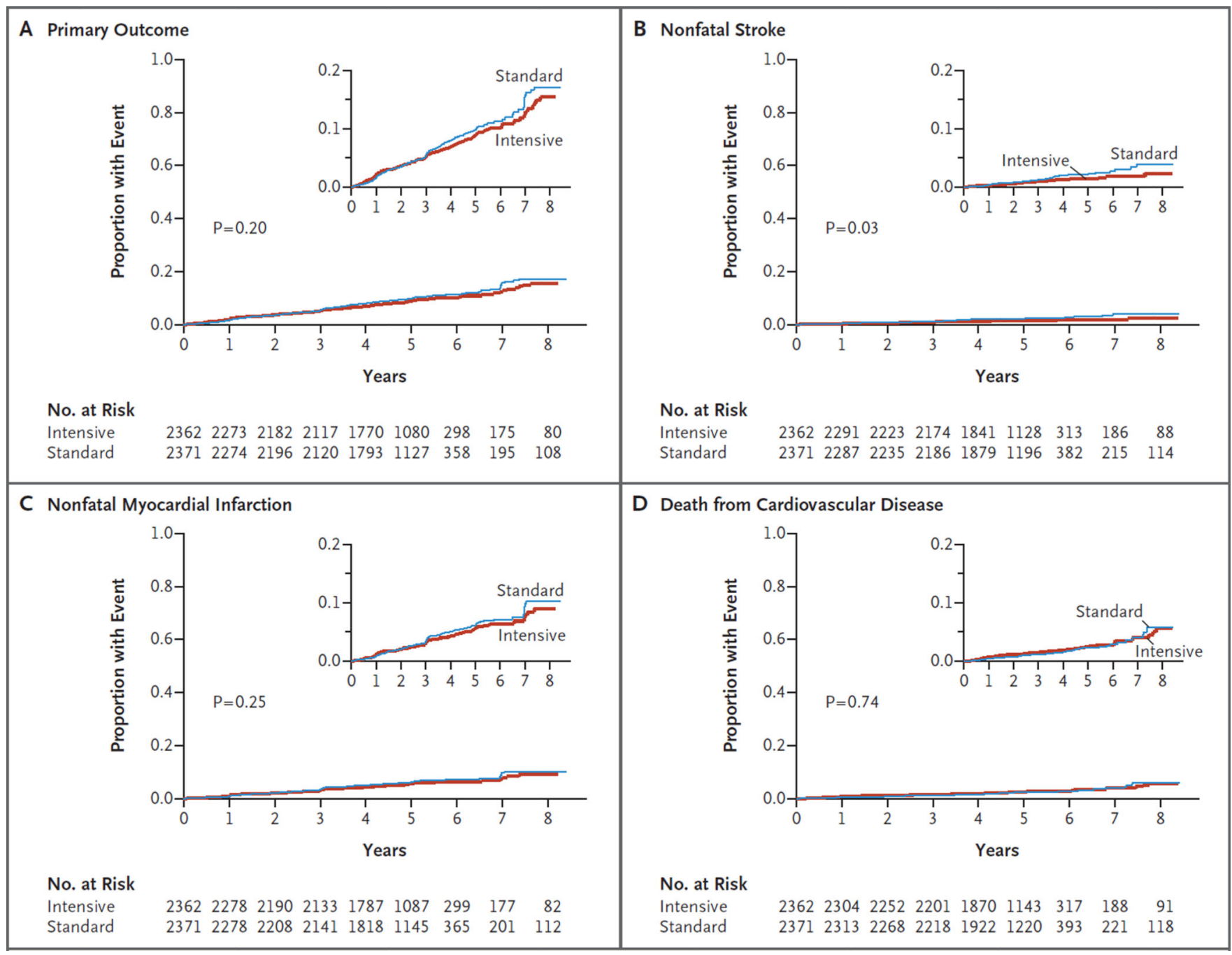

Figure 2. Kaplan-Meier Analyses of Selected Outcomes

Shown are the proportions of patients with events for the primary composite outcome (Panel A) and for the individual components of the primary outcome (Panels B, C, and D). The insets show close-up versions of the graphs in each panel. 


\section{Table 1}

Characteristics of the Participants at Baseline. ${ }^{*}$

\begin{tabular}{|c|c|c|c|c|}
\hline Characteristic & $\begin{array}{c}\text { Overall } \\
(\mathbf{N}=\mathbf{4 7 3 3})\end{array}$ & $\begin{array}{c}\text { Intensive Therapy } \\
\qquad(\mathbf{N}=\mathbf{2 3 6 2})\end{array}$ & $\begin{array}{c}\text { Standard Therapy } \\
(\mathbf{N}=\mathbf{2 3 7 1})\end{array}$ & P Value \\
\hline Age $-\mathrm{yr}$ & $62.2 \pm 6.9$ & $62.2 \pm 6.8$ & $62.2 \pm 6.9$ & 0.82 \\
\hline Female sex- no. $(\%)$ & $2258(47.7)$ & $1128(47.8)$ & $1130(47.7)$ & 0.95 \\
\hline \multicolumn{5}{|l|}{ Race or ethnic group - no. $(\%)^{\dagger}$} \\
\hline Non-Hispanic white & $2864(60.5)$ & 1455 (61.6) & $1409(59.4)$ & 0.13 \\
\hline Black & $1142(24.1)$ & $561(23.8)$ & $581(24.5)$ & 0.56 \\
\hline Hispanic & $330(7.0)$ & $159(6.7)$ & $171(7.2)$ & 0.53 \\
\hline Education - no./total no. $(\%)$ & & & & 0.18 \\
\hline Less than high school & $771 / 4729(16.3)$ & $404 / 2359(17.1)$ & $367 / 2370(15.5)$ & \\
\hline High-school graduate or GED & $1271 / 4729(26.9)$ & $606 / 2359(25.7)$ & $665 / 2370(28.1)$ & \\
\hline Some college & $1530 / 4729(32.4)$ & $776 / 2359(32.9)$ & $754 / 2370(31.8)$ & \\
\hline College degree or higher & $1157 / 4729(24.5)$ & $573 / 2359(24.3)$ & $584 / 2370(24.6)$ & \\
\hline Previous cardiovascular event- no. (\%) & $1593(33.7)$ & $804(34.0)$ & $789(33.3)$ & 0.58 \\
\hline Previous heart failure - no./total no. (\%) & $203 / 4683(4.3)$ & $109 / 2338(4.7)$ & $94 / 2345(4.0)$ & 0.28 \\
\hline Cigarette-smoking status - no./total no. $(\%)$ & & & & 0.94 \\
\hline Current & $626 / 4728(13.2)$ & $314 / 2358(13.3)$ & $312 / 2370(13.2)$ & \\
\hline Former & $1981 / 4728(41.9)$ & $992 / 2358(42.1)$ & $989 / 2370(41.7)$ & \\
\hline Never & $2121 / 4728(44.9)$ & $1052 / 2358(44.6)$ & $1069 / 2370(45.1)$ & \\
\hline Weight $-\mathrm{kg}$ & $92.0 \pm 18.6$ & $92.1 \pm 19.4$ & $91.8 \pm 17.7$ & 0.57 \\
\hline Body-mass index & $32.1 \pm 5.6$ & $32.2 \pm 5.7$ & $32.1 \pm 5.4$ & 0.58 \\
\hline \multicolumn{5}{|l|}{ Blood pressure $-\mathrm{mm} \mathrm{Hg}^{\ddagger}$} \\
\hline \multicolumn{5}{|l|}{ All participants } \\
\hline Systolic & $139.2 \pm 15.8$ & $139.0 \pm 16.1$ & $139.4 \pm 15.5$ & 0.47 \\
\hline Diastolic & $76.0 \pm 10.4$ & $75.9 \pm 10.6$ & $76.0 \pm 10.2$ & 0.87 \\
\hline \multicolumn{5}{|l|}{ Participants taking no medication at screening } \\
\hline Systolic & $139.4 \pm 14.3$ & $139.8 \pm 15.0$ & $139.1 \pm 13.7$ & 0.53 \\
\hline Diastolic & $77.5 \pm 9.4$ & $77.5 \pm 9.5$ & $77.4 \pm 9.4$ & 0.86 \\
\hline \multicolumn{5}{|c|}{ Participants taking at least one medication at screening } \\
\hline Systolic & $139.2 \pm 16.0$ & $138.9 \pm 16.3$ & $139.4 \pm 15.8$ & 0.34 \\
\hline Diastolic & $75.7 \pm 10.5$ & $75.7 \pm 10.7$ & $75.8 \pm 10.3$ & 0.87 \\
\hline Duration of diabetes $-\mathrm{yr}$ & & & & 0.86 \\
\hline Median & 10 & 9 & 10 & \\
\hline Interquartile range & $5-15$ & $5-15$ & $5-15$ & \\
\hline Glycated hemoglobin — \% & $8.3 \pm 1.1$ & $8.4 \pm 1.1$ & $8.3 \pm 1.1$ & 0.08 \\
\hline Fasting plasma glucose $-\mathrm{mg} / \mathrm{dl}$ & $174.7 \pm 57.7$ & $176.1 \pm 57.7$ & $173.2 \pm 57.7$ & 0.09 \\
\hline \multicolumn{5}{|l|}{ Cholesterol $-\mathrm{mg} / \mathrm{dl}$} \\
\hline Total & $192.8 \pm 44.7$ & $194.1 \pm 45.1$ & $191.4 \pm 44.3$ & 0.04 \\
\hline
\end{tabular}




\begin{tabular}{|lcccc|}
\hline Characteristic & $\begin{array}{c}\text { Overall } \\
(\mathbf{N}=\mathbf{4 7 3 3})\end{array}$ & $\begin{array}{c}\text { Intensive Therapy } \\
(\mathbf{N}=\mathbf{2 3 6 2})\end{array}$ & $\begin{array}{c}\text { Standard Therapy } \\
(\mathbf{N = 2 3 7 1})\end{array}$ & P Value \\
\hline Low-density lipoprotein & $110.0 \pm 36.7$ & $111.1 \pm 37.4$ & $108.8 \pm 36.0$ & 0.03 \\
\hline High-density lipoprotein & & & & \\
\hline Women & $51.3 \pm 13.8$ & $51.3 \pm 13.4$ & $51.3 \pm 14.3$ & 0.99 \\
\hline Men & $41.7 \pm 11.8$ & $41.4 \pm 11.2$ & $42.0 \pm 12.4$ & 0.17 \\
\hline Plasma triglycerides $-\mathrm{mg} / \mathrm{dl}$ & & & 147 & 0.71 \\
\hline Median & 147 & $98-224$ & \\
\hline Interquartile range & $98-226$ & $98-227$ & $4.5 \pm 0.8$ & 0.73 \\
\hline Potassium $-\mathrm{mg} / \mathrm{dl}$ & $4.5 \pm 0.7$ & $4.5 \pm 0.5$ & $0.9 \pm 0.2$ & 0.98 \\
\hline Serum creatinine $-\mathrm{mg} / \mathrm{dl}$ & $0.9 \pm 0.2$ & $0.9 \pm 0.2$ & $91.7 \pm 27.1$ & 0.93 \\
\hline Estimated GFR $-\mathrm{ml} / \mathrm{min} / 1.73 \mathrm{~m}^{2}$ & $91.6 \pm 28.8$ & $91.6 \pm 30.3$ & & 0.64 \\
\hline Ratio of urinary albumin $(\mathrm{mg})$ to creatinine $(\mathrm{g})$ & & & 14.0 & \\
\hline Median & 14.3 & 14.6 & $6.9-45.8$ & \\
\hline Interquartile range & $6.9-44.8$ & $7.0-43.7$ & & \\
\hline
\end{tabular}

* Plus-minus values are means +SD. Percentages may not total 100 because of rounding. To convert the values for glucose to millimoles per liter, multiply by 0.055551 . To convert the values for cholesterol to millimoles per liter, multiply by 0.02586 . To convert the values for triglycerides to millimoles per liter, multiply by 0.01129 . To convert the values for potassium to millimoles per liter, multiply by 0.2558 . To convert the values for creatinine to micromoles per liter, multiply by 88.4 . GED denotes general equivalency diploma, and GFR glomerular filtration rate.

${ }^{\dagger}$ Race or ethnic group was self-reported, and participants could check multiple categories.

*Data were available for 4733 participants in the total cohort, 599 who were taking no medication at screening and 4134 who were taking one or more medications at screening. 
Table 2

Serious Adverse Events and Clinical Measures after Randomization. ${ }^{*}$

\begin{tabular}{|c|c|c|c|}
\hline Variable & $\begin{array}{l}\text { Intensive Therapy } \\
(\mathbf{N}=\mathbf{2 3 6 2})\end{array}$ & $\begin{array}{l}\text { Standard Therapy } \\
(\mathbf{N}=\mathbf{2 3 7 1})\end{array}$ & P Value \\
\hline \multicolumn{4}{|l|}{ Serious adverse events - no. $(\%)^{\dagger}$} \\
\hline Event attributed to blood-pressure medications & $77(3.3)$ & $30(1.27)$ & $<0.001$ \\
\hline Hypotension & $17(0.7)$ & $1(0.04)$ & $<0.001$ \\
\hline Syncope & $12(0.5)$ & $5(0.21)$ & 0.10 \\
\hline Bradycardia or arrhythmia & $12(0.5)$ & $3(0.13)$ & 0.02 \\
\hline Hyperkalemia & $9(0.4)$ & $1(0.04)$ & 0.01 \\
\hline Angioedema & $6(0.3)$ & $4(0.17)$ & 0.55 \\
\hline Renal failure & $5(0.2)$ & $1(0.04)$ & 0.12 \\
\hline End-stage renal disease or need for dialysis & $59(2.5)$ & $58(2.4)$ & 0.93 \\
\hline \multicolumn{4}{|c|}{ Symptoms affecting quality of life - no./total no. $(\%)^{\ddagger}$} \\
\hline Hives or swelling & $44 / 501(8.8)$ & $41 / 468(8.8)$ & 1.00 \\
\hline Dizziness when standing & $217 / 501(44.3)$ & $188 / 467(40.3)$ & 0.36 \\
\hline \multicolumn{4}{|l|}{ Adverse laboratory measures - no. (\%) } \\
\hline Potassium $<3.2 \mathrm{mmol} /$ liter & $49(2.1)$ & $27(1.1)$ & 0.01 \\
\hline Potassium $>5.9 \mathrm{mmol} /$ liter & $73(3.1)$ & $72(3.0)$ & 0.93 \\
\hline \multicolumn{4}{|l|}{ Elevation in serum creatinine } \\
\hline$>1.5 \mathrm{mg} / \mathrm{dl}$ in men & $304(12.9)$ & $199(8.4)$ & $<0.001$ \\
\hline$>1.3 \mathrm{mg} / \mathrm{dl}$ in women & $257(10.9)$ & $168(7.1)$ & $<0.001$ \\
\hline Estimated GFR $<30 \mathrm{ml} / \mathrm{min} / 1.73 \mathrm{~m}^{2}$ & $99(4.2)$ & $52(2.2)$ & $<0.001$ \\
\hline \multicolumn{4}{|l|}{ Clinical measures $f$} \\
\hline Glycated hemoglobin - \% & $7.6 \pm 1.3$ & $7.5 \pm 1.2$ & 0.13 \\
\hline Fasting plasma glucose $-\mathrm{mg} / \mathrm{dl}$ & $147.1 \pm 56.6$ & $148.1 \pm 57.5$ & 0.58 \\
\hline Plasma LDL cholesterol — mg/dl & $98.7 \pm 40.3$ & $96.8 \pm 37.8$ & 0.10 \\
\hline Plasma HDL cholesterol — mg/dl & $46.7 \pm 14.0$ & $47.8 \pm 14.9$ & 0.02 \\
\hline Plasma triglycerides $-\mathrm{mg} / \mathrm{dl}$ & & & 0.001 \\
\hline Median & 138 & 131 & \\
\hline Interquartile range & $97-210$ & $92-197$ & \\
\hline Potassium - mg/dl & $4.3 \pm 0.5$ & $4.4 \pm 0.5$ & 0.17 \\
\hline Serum creatinine $-\mathrm{mg} / \mathrm{dl}$ & $1.1 \pm 0.4$ & $1.0 \pm 0.5$ & $<0.001$ \\
\hline Estimated GFR $-\mathrm{ml} / \mathrm{min} / 1.73 \mathrm{~m}^{2}$ & $74.8 \pm 25.0$ & $80.6 \pm 24.8$ & $<0.001$ \\
\hline Ratio of urinary albumin (mg) to creatinine $(\mathrm{g})$ & & & $<0.001$ \\
\hline Median & 12.6 & 14.9 & \\
\hline Interquartile range & $6.4-1.7$ & $7.0-56.8$ & \\
\hline Microalbuminuria - no./total no. $(\%)$ & $656 / 2174(30.2)$ & $712 / 2205(32.3)$ & 0.13 \\
\hline Macroalbuminuria - no. /total no. (\%) & $143 / 2174(6.6)$ & $192 / 2205(8.7)$ & 0.009 \\
\hline
\end{tabular}




\begin{tabular}{|lccc|}
\hline Variable & $\begin{array}{c}\text { Intensive Therapy } \\
(\mathbf{N}=\mathbf{2 3 6 2})\end{array}$ & $\begin{array}{c}\text { Standard Therapy } \\
(\mathbf{N}=\mathbf{2 3 7 1})\end{array}$ & P Value \\
\hline Serious adverse events - no. $(\%)^{\dagger}$ & & & \\
\hline Weight $-\mathrm{kg}$ & $93.3 \pm 21.2$ & $92.5 \pm 20.2$ & 0.20 \\
\hline
\end{tabular}

* Plus-minus values are means +SD. To convert the values for glucose to millimoles per liter, multiply by 0.055551 . To convert the values for cholesterol to millimoles per liter, multiply by 0.02586 . To convert the values for triglycerides to millimoles per liter, multiply by 0.01129 . To convert the values for potassium to millimoles per liter, multiply by 0.2558 . To convert the values for creatinine to micromoles per liter, multiply by 88.4. GFR denotes glomerular filtration rate, HDL high-density lipoprotein, and LDL low-density lipoprotein.

${ }^{\dagger}$ Serious adverse events are events that are life-threatening, cause permanent disability, or necessitate hospitalization (see Section 7 in Supplementary Appendix 1).

Fymptoms were assessed at 12, 36, and 48 months after randomization in a random sample of 969 participants who were assessed for healthrelated quality of life.

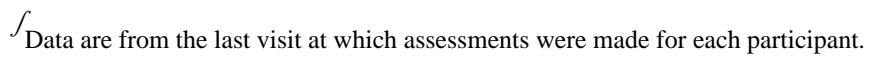


